\title{
SIMULATION OF EGNOS SATELLITE NAVIGATION SIGNAL USAGE FOR AIRCRAFT LPV PRECISION INSTRUMENT APPROACH
}

\author{
Michal HVEZDA (1D* \\ Institute of Aerospace Engineering, Brno University of Technology, Brno, Czech Republic
}

Received 1 June 2020; accepted 27 November 2020

\begin{abstract}
Satellite navigation has become a very important topic in the air transport industry along with its application in instrument approach procedures. Recently, extracted statistical characteristics of the European Geostationary Navigation Overlay Service (EGNOS) satellite signal have been made available from real measurements in the Czech Republic. The numerical modeling approach is taken for a feasibility study of automatic aircraft control during the Localizer Performance with Vertical Guidance (LPV) precision approach based on such navigation data. The model incorporates Kalman filtering of the stochastic navigation signal, feed-back control of L-410 aircraft dynamics and the calculation of approach progress along the predefined procedure. Evaluation of the performance of the system prototype is performed using the scenarios developed with a strong interest in altitude control. The specific scenario is focused on a curved approach which offers a huge advantage of the approaches based on the Satellite-based Augmentation System (SBAS) compared to ones with the Instrument Landing System (ILS). Outputs of simulation executions are statistically analyzed and assessed against predefined navigation performance goals equivalent to ILS categories with a positive outcome.
\end{abstract}

Keywords: EGNOS, LPV approach, aircraft control, autopilot, performance-based navigation, modelling, Kalman filtering, simulation, prototype.

\section{Introduction}

Many European airports now use satellites as navigation aids for the approach of aircraft (Vencius, 2013). The signal itself as provided by satellites does not have sufficient navigation performance parameters to enable safe aircraft operation during approaches (ICAO, 2012). The focus of approach procedure designers is going in the direction of satellite augmentation systems, ground or satellite based, particularly to use advantages of European Geostationary Navigation Overlay Service (EGNOS) in Europe.

This is also true in the Czech Republic where Localizer Performance with Vertical Guidance (LPV) procedures are in operation or planned on the runways of most airports with international transport. Current LPV procedures reach equivalence with ILS CAT I approaches once decision heights are at the $60 \mathrm{~m}(200 \mathrm{ft})$ level, however statistical analysis of real measurements of the EGNOS navigation signal in this area shows huge potential for even wider use of the signal, so it is being studied across the European Union (Vassilev \& Vassileva, 2012; Grunwald et al., 2019).
The European Global Navigation Satellite Systems Agency reports 460 operational approach procedures using the navigation signal provided by the EGNOS (EGNSSA, 2020). The opportunities to use the signal for aviation purposes are currently investigated for the geographical locations on the EGNOS coverage edge (Beldjilali et al., 2020). The data from the experiments are available from the measurements made using the onboard sensors during the landing of the aircraft (Krasuski \& Wierzbicki, 2020). These characteristics of the navigational signal can be further used in the simulations involving aircraft mechanics and onboard systems. Such simulations may help to explore the area of usability of the EGNOS system as a provider of the inputs for the aircraft navigation and control systems used during the execution of approach procedures.

This feasibility study, based on the model-based development (MBD) approach commonly used within the aviation industry (Scilab, 2020), attempts to develop a model of the aircraft control system during the approach. The system is fed by the EGNOS navigation signal and operates within a framework of precision approach below

${ }^{*}$ Corresponding author. E-mail: michal.hvezda@vut.cz 
decision heights of existing LPV approaches. The aim is to exercise the possibilities of the navigation signal and prove its usability via utilization of the developed system functional prototype. Such simulation efforts can effectively exercise some aspects of planned aircraft operations which otherwise have to be validated by costly trial flights (Fellner, 2011).

\section{Architecture of the system proposed for evaluation}

Evaluation of controlled aircraft performance is simplified by avoiding complex human behaviour so that the developed model is a model of the automated system as presented in Figure 1. The control system involves two parts: (1) navigation filter modules, and (2) controller modules. Other modules are involved to ensure that the task of the evaluation of the design of the aircraft approach automated system based on the EGNOS navigation signal is completely sufficient. The flight mechanics is represented by the aircraft dynamics and by the aircraft kinematics. The inputs of the module of the dynamics are connected to the outputs of the controller which creates a closed loop simulation. The dynamics is reflected by the kinematics adjusted to the approach flight phase. Sensors (especially an EGNOS navigation one) provide simulation of the data necessary for the navigation filter and for the controller.

Such a modelling approach creates a simulation which is an alternative to other approaches (Antemijczuk et al., 2012). Properties of the proposed solution is that it covers all necessary functional modules at appropriate complexity levels. Such a closed loop simulation is consistent and complete. These properties also ensure that the functionality of the simulation can be easily verified.

\subsection{Modelled avionics}

The filter module provides the position of the aircraft based on input signals measured by satellite navigation sensors. The Kalman filter (Sorenson, 1985) is used within

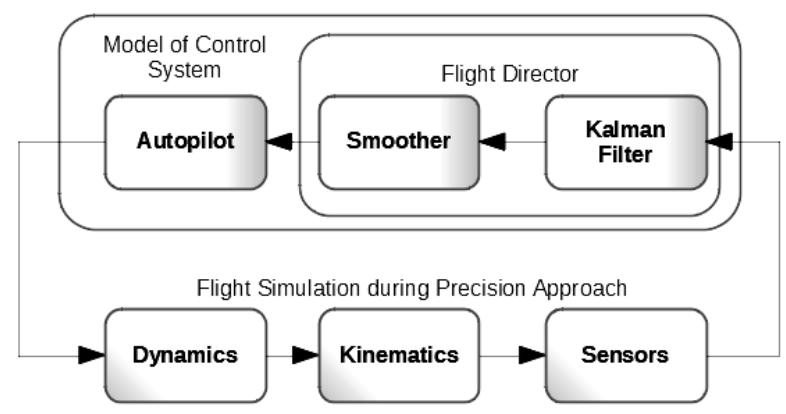

Figure 1. Modules and corresponding signal flow of the modelled system and processes the model to estimate the aircraft position while incorporating measurements with the gain of:

$$
K=\frac{S_{n \mid n-1} \cdot H^{\prime}}{H \cdot S_{n \mid n-1} \cdot H^{\prime}+R}
$$

where:

$S_{n \mid n-1}$ is the covariance matrix of the estimated filter state errors derived from the previous filtration step;

$H$ is the matrix representing projection from the filter state space to the measurement space, and

$R$ is the measurement error covariance matrix.

Filter input is the difference of the actual and planned position of the aircraft and the estimated states are again the difference and its change, so that:

$$
H=\left[\begin{array}{ll}
1 & 0
\end{array}\right] \text {. }
$$

An important feature of the filter is that it can continue in its function even when measurements are not provided, e.g. because of signal dropout. This operation mode is covered by the model and is incorporated in the set of verification scenarios discussed below. Estimated filter states are updated as follows:

$$
x_{n \mid n-1}=F \cdot x_{n-1 \mid n-1},
$$

where:

$x_{n-1 \mid n-1}$ is the vector of the estimated filter states in the previous filtration step;

$x_{n \mid n-1}$ is the vector of the estimated filter states in the current step, and

$F$ is the matrix representing the evolution of the inner filter model.

The best estimation functionality of the filter is achieved when, in this case, the inner system is a linear approximation of convoluted behavior of aircraft mechanics and its automated controller. The model developed within this work is sufficiently robust to enable such analysis, design and configuration. However, such a detailed focus on the inner model is beyond the scope of this work. Instead, any unknowns in the behavior of the whole system in loop-back as seen from the filter perspective are represented by the noise covariance matrix of the inner filter system. Then, the solution proposed for the evaluated system is expressed as:

$$
\begin{aligned}
& x_{n-1 \mid n-1}=\left[\begin{array}{c}
(\Delta x)_{E} \\
(\Delta y)_{E}
\end{array}\right] ; \\
& (\Delta y)_{E}=\left(\frac{d(\Delta x)}{d t}\right)_{E} ; \\
& F=\left[\begin{array}{cc}
1 & \Delta t \\
0 & 1
\end{array}\right] ; \\
& S_{n \mid n-1}=F \cdot S_{n-1 \mid n-1} \cdot F^{\prime}+Q,
\end{aligned}
$$

where:

$(x)_{E}$ is the estimation of $\mathrm{x}$ based on its measurements; $\Delta x$ is the difference of the actual and planned position of the aircraft, $\Delta t$ is the time period between filtering steps; 
$S_{n-1 \mid n-1}$ is the covariance matrix of estimated filter state errors derived from the previous filtration step, and $Q$ is the noise covariance matrix representing errors caused by the description of the controlled aircraft dynamics using linear approximation described by matrix $F$ only.

A smoother (Meloun \& Militky, 2002) module is introduced into the model to support full flight director functionality. Discrete data provided by the EGNOS system and filtered by the digital Kalman filter need to be applied in the field of consequent continuous control logic. The smoother is designed as a sextic spline which ensures that both the position and its derivative are continuous in time. Such a requirement is necessary not only to ensure that the controller operation is sufficient but it's more important with respect to the aircraft control surfaces being loaded by the filtered signal. More details are provided in Appendix A.

The results of the functional operation of the filter and smoother are demonstrated in Figure 2. Decreased variance within the data after filtering is observable along with the delay in response to input change as an essential part of the smoothening process.

The autopilot controller has configurable parameters and its architecture and features are further discussed along with solutions of navigation cases. It corresponds to the method of searching for the optimal gain and time constant of controller components to achieve a good balance between the reaction speed and the overshooting resulting in the controlled aircraft position.

\subsection{Simulated processes}

The essential part of the sensor simulation is to provide the EGNOS navigation signal as an input of the Kalman navigation filter. The principle is to apply known statistical characteristics of the data to the current simulated position of the aircraft. The characteristics are shaped as stochastic noise with Gaussian distribution which is pre-generated based on the following parameters (Ptacek, 2014):

- Mean of $0.30 \mathrm{~m}$ in altitude;

- Standard deviation of $0.48 \mathrm{~m}$ in altitude;

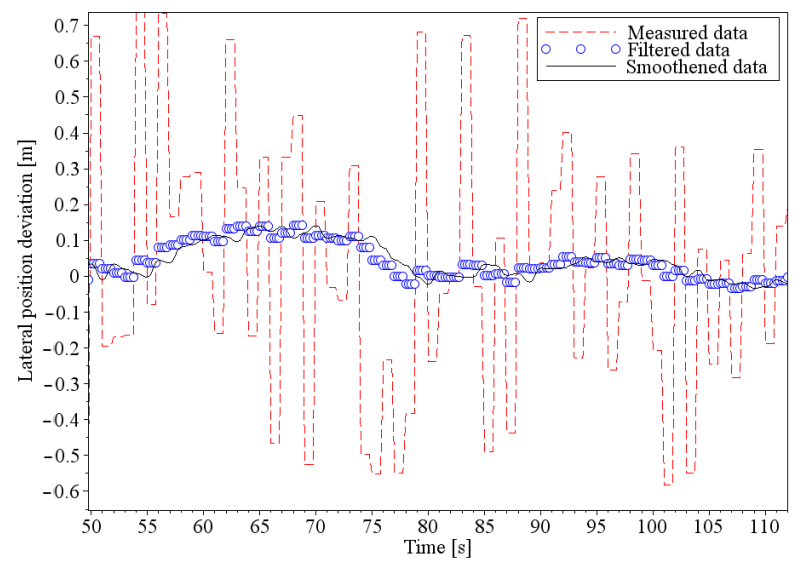

Figure 2. Filtration and smoothening effects on EGNOS navigation signal samples. Part of the approach process shown
- Mean of $0.65 \mathrm{~m}$ in the north - south direction used for lateral position;

- Convolution of standard deviation of $0.30 \mathrm{~m}$ in the north - south direction and of the standard deviation of $0.26 \mathrm{~m}$ in the east - west direction;

- Discretization period of $1 \mathrm{~s}$.

These characteristics provide specific values of Kalman filter settings for matrix $R$ and sample period $\Delta t$. The matrix is degraded to scalar only once the position is measured in either a lateral or vertical experiment configuration. Also:

$$
\Delta t=1 \text {. }
$$

In the presented model, the actual position of the aircraft is impacted by multiple simulated factors. These are 1) aircraft dynamics, 2) approach procedure, and 3) environmental conditions.

The regular way of simulation of aircraft dynamics (Cook, 2012) is in six degrees of freedom as described for forces and moments within aircraft body coordinates:

$$
\begin{aligned}
& m(\dot{U}-V R+W Q)=X_{a}+X_{g}+X_{e}+X_{p}+X_{d} ; \\
& m(\dot{V}+U R-W P)=Y_{a}+Y_{g}+Y_{e}+Y_{p}+Y_{d} ; \\
& m(\dot{W}-U Q+V P)=Z_{a}+Z_{g}+Z_{e}+Z_{p}+Z_{d} ; \\
& I_{x x} \dot{P}-\left(I_{y y}-I_{z z}\right) R Q-I_{x z}(P Q+\dot{R})=L_{a}+L_{g}+L_{e}+L_{p}+L_{d},
\end{aligned}
$$

$I_{y y} \dot{Q}+\left(I_{x x}-I_{z z}\right) P R+I_{x z}\left(P^{2}-R^{2}\right)=M_{a}+M_{g}+M_{e}+M_{p}+M_{d}$,

$I_{z z} \dot{R}-\left(I_{x x}-I_{y y}\right) P Q+I_{x z}(Q R-\dot{P})=N_{a}+N_{g}+N_{e}+N_{p}+N_{d}$,

where:

$m$ is the aircraft weight;

$I_{x x}, I_{y y}, I_{z z}, I_{x z}$ are elements of tensor of moments of inertia;

$U, V, W$ are elements of vector of aircraft body speed;

$P, Q, R$ are elements of vector of aircraft body angular speed;

$X_{X}, Y_{X}, Z_{X}$ are elements of force vector caused by origin $\mathrm{X}$, and

$L_{Y}, M_{Y}, N_{Y}$ are elements of vector of momentum caused by origin $\mathrm{Y}$.

Indices of right-hand side operands of equations (9) to (14) designate their origin: $a$ - aerodynamic, $g$ - gravitational, $e-$ control surfaces, $p$ - propulsion, $d-$ atmospheric. However, the 6-degree of freedom simulation is not used to limit the scope of the work and keep the focus on navigation data processing.

The aim to examine the navigation data using the method of functional prototype experiments leads to the selection of the aircraft. The L-410 seems to be the best option when considering factors such as aircraft speed category (ICAO, 2009), availability of dynamic models, and its common use in the Czech Republic. Dynamics are 
re-used (CTU, 2015) and integrated as a linear state space system, i. e.:

$$
\begin{aligned}
& \dot{x}=A \cdot x+B \cdot u ; \\
& y=C \cdot x+D \cdot u,
\end{aligned}
$$

where:

$x$ is the state vector;

$u$ is the control vector;

$y$ is the output vector of the system;

and matrices $A$ and $B$ are taken from the L-410 aircraft model.

Lateral movement dynamics use the following states and controls:

$$
\begin{aligned}
& x=\{\beta, \gamma, \psi, \dot{\gamma}, \dot{\psi}\}^{\prime} ; \\
& u=\{\xi, \varsigma\}^{\prime} .
\end{aligned}
$$

Movement in the longitudinal direction is represented as:

$$
\begin{aligned}
& x=\{\Delta v, \alpha, \vartheta, \dot{\vartheta}\}^{\prime} ; \\
& u=\{\eta, \tau\}^{\prime} .
\end{aligned}
$$

The kinematics of the aircraft is implemented within the coordinate frame determined by the approach procedure. The process defining operation of the aircraft studied herein follows the operation of the ILS system. The coordinate system is defined by localizer and glide slope planes as illustrated in Figure 3. The progress of aircraft approach is represented by the decrease of aircraft distance from the runway along the approach axis. The axis is an intersection of the planes. The approach process defines the expected height for the given runway distance per predefined glide path angle. States of the aircraft dynamics are used directly as kinematic simulation inputs so that:

$$
\begin{aligned}
& C=I ; \\
& D=0,
\end{aligned}
$$

for the matrices from equation (16).

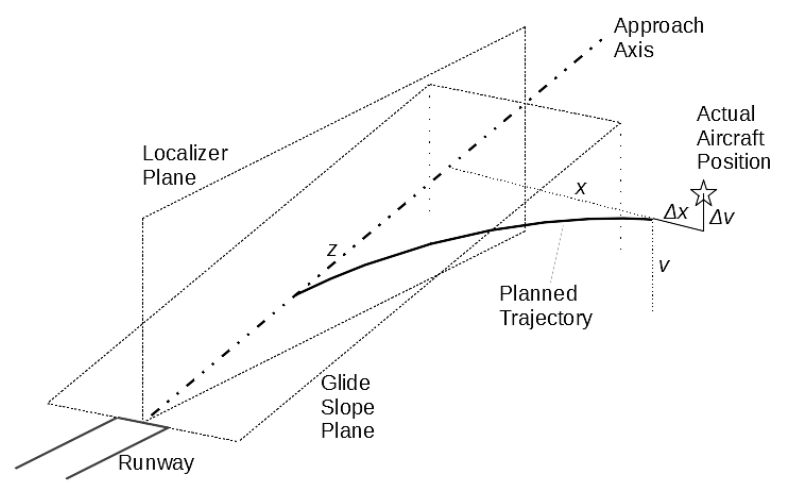

Figure 3. Established coordinate system based on the localizer and glide slope planes defined by the ILS system. The system is enhanced to allow to definition of the curved trajectory as functions of distance from the localizer plane $(x)$ and height $(v)$ on distance from the runway $(z)$. Actual aircraft position is referenced to the planned position by differences $\Delta x$ and $\Delta v$ in lateral and vertical directions respectively
The solution of the task adopted in this work should also fulfil the regular ILS operation which can be expressed by the definition of the approach windows per the ILS categories in Table 1.

Table 1. Definition of the windows at a height of $15.24 \mathrm{~m}$ (50 ft) above runway per precision approach categories (ICAO, 2016)

\begin{tabular}{|l|c|c|c|}
\hline $\begin{array}{c}\text { Direction / } \\
\text { Precise Approach } \\
\text { Category }\end{array}$ & CAT I & CAT II & CAT III \\
\hline Horizontal & $\begin{array}{c} \pm 10.668 \mathrm{~m} \\
(35 \mathrm{ft})\end{array}$ & $\begin{array}{c} \pm 7.620 \mathrm{~m} \\
(25 \mathrm{ft})\end{array}$ & $\begin{array}{c} \pm 6.096 \mathrm{~m} \\
(20 \mathrm{ft})\end{array}$ \\
\hline Vertical & \multicolumn{3}{|c|}{ $\pm 3.048 \mathrm{~m}(10 \mathrm{ft})$} \\
\hline
\end{tabular}

\subsection{Parametrization of the system}

The views on the top level of the system implementation for lateral and longitudinal are presented in Appendix B. Execution of the simulation will not be possible without an appropriate parametrization of the system. The system contains a set of configurable parameters which are set by the Scilab script during the initialization of the model for the simulation execution. The parameters are:

- Statistical characteristics of the navigation signal;

- Elements of the noise matrix of the inner Kalman filter system;

- Coefficients of the proportional, derivative and integration components of the controller;

- Size and source file name of the stochastically generated errors of the navigation signal measurements.

These parameters (except the ones related to the navigation signal) were experimentally determined during simulation executions with the help of system identification methods (Balate, 2003).

\section{Comparison to the Instrument Landing System}

The definition of the approach process on the ILS system functionality (ICAO, 2016) establishes a platform to recognize the advantages of the LPV approaches. These approaches, compared to ILS ones, reside on performancebased navigation (PBN). The decision height for $3 \mathrm{D}$ satellite-based augmentation system (SBAS) approaches (ICAO, 2018) can be determined based on the specific navigation signal performance in the airport area along with the PBN philosophy of giving responsibility of navigation aid selection to the pilot. Such new options help to decrease the number of missed approaches.

While ILS approaches support the definition of procedures along the approach axis only, the LPV procedures can be defined using a curve. This brings the advantage of using them for runways where ILS cannot be used due to minimum obstacle clearance (MOC) constraints (ICAO, 2020). The ability of LPV to define safe procedures per $\mathrm{PBN}$ rules provides an opportunity to use satellite navigation for vertical guidance during approach flight phases. It has a positive impact on the flow and capacity of air 
transport for the airports and may improve environmental conditions, e.g. noise reduction in the concerned area (EGNOS, 2002).

Evaluation of the proposed model takes into consideration both new and existing scenarios. The advantage of the LPV approach along the predefined curve is specifically verified as discussed below. An extensive focus is given to model operation in the scenarios existing with ILS so that a sufficient LPV performance is demonstrated in altitude control. Furthermore, objectives for the definition of the whole set of verification scenarios are also provided.

\subsection{Approach using the predefined curve}

The curve for a planned approach is defined as a function of the distance from the approach axis within the glide slope plane on the runway distance measured along the axis. In such a way, planes defined by the ILS system are used for the description of the hyperbolic curve. An additional control signal besides the signal from the navigation filter is derived from known recipe of the curve which is expressed as:

$$
\frac{(x-n)^{2}}{a^{2}}-\frac{\left[\left(z_{F A F}-z\right)-m\right]^{2}}{\left(\frac{a}{\tan \psi_{0}}\right)^{2}}=1
$$

where:

$x$ is the lateral position of the aircraft as illustrated in Figure 3; $n$ is the offset of hyperbolic centre with respect to the localizer plane;

$a$ is the axis of the hyperbolic curve;

$z_{F A F}$ is the distance of the final approach fix from the runway;

$z$ is the distance of the aircraft from the runway as illustrated in Figure 3;

$m$ is the offset of the hyperbolic curve centre along the approach axis;

$\psi_{0}$ is the direction of the hyperbolic curve asymptote which represents aircraft yaw.

The tangent of the curve in the planned position of the aircraft on the curve of $z_{0}$ and $x_{0}$ is:

$x=\frac{1}{x_{0}-n}\left[a^{2}+\left(\tan \psi_{0}\right)^{2}\left[\left(z_{F A F}-z\right)-m\right]\left[\left(z_{F A F}-z_{0}\right)-m\right]\right]+n$.

The signal is constructed based on:

$$
\dot{\psi}_{c}=\frac{d\left[-\tan ^{-1}\left(\frac{d x}{d z}\right)\right]}{d t},
$$

which represents the change of the yaw angle along the curve. The property of the selected hyperbolic relation is that the signal is very close to the constant function which has a stabilizing impact on the aircraft dynamics.

Initialization and transition effects can be seen in Figure 4. Initialization effects are present in the developed level of the simulation which does not fully address the

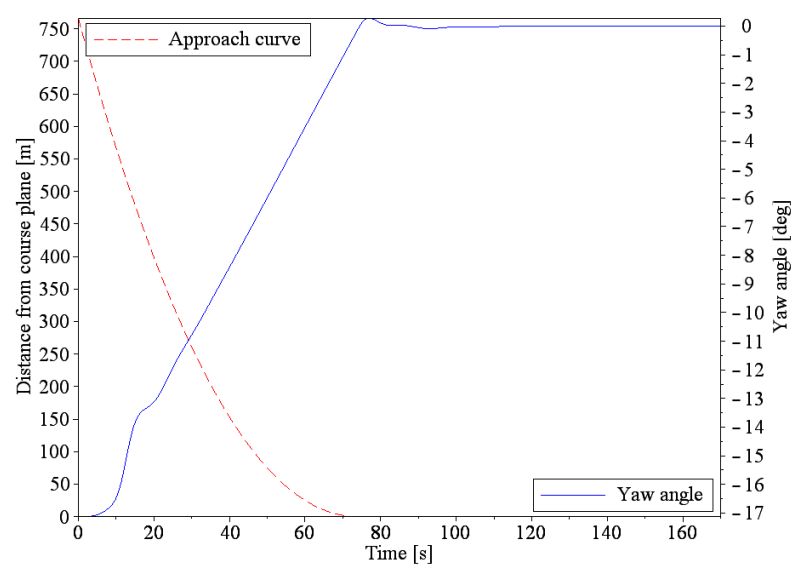

Figure 4. Change of the yaw angle during approach along the defined curve

trimming of the aircraft dynamics. Implementation of trimming was not included into the scope of the work. The transition effect is caused by the step change of the second derivative of the planned trajectory in the merging point of hyperbola into the approach axis. Simple smoothening and feedback are added into the control signal to fulfil the requirements expected on the lateral precision of the aircraft.

Values used for the presented experiment are:

$$
\begin{aligned}
& \psi_{0}=35 \frac{\pi}{180} \\
& a=0.7 z_{F A F} \\
& m=0.7 a \\
& n=-a
\end{aligned}
$$

\subsection{Flight control in altitude}

The role of the control system (Balate, 2003) is to cover flight control in the horizontal direction and in altitude within various conditions encompassing range of approach angles and the impact of the front wind of a wide set of magnitudes.

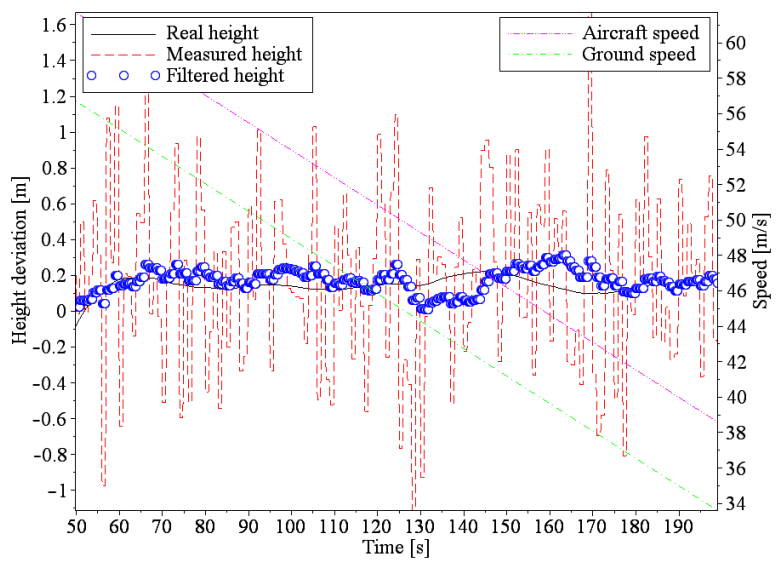

Figure 5. Approach control in altitude within the head wind scenario. Ramp-up effects during system initialization are not shown 
The system must support the decrease of the aircraft speed along the path as well. The speed change impacts abilities of the controller function which must be adjusted to support the whole speed range and the speed change. A general controller design composed of the proportional and derivative components (PD controller) is expanded to also include an integration component (PID one). An example of the model operation is presented in Figure 5.

\subsection{Set of verification scenarios}

Motivation for the study of the environmental conditions of the approach process controlled by the proposed model is to verify the model design by the set of possible use case scenarios. Deployment of a new avionic system within the industry is a complex and sophisticated process, e.g. RTCA recommendations for the system, hardware and software (RTCA, 2012) are used for the certification of avionic systems. Although the application of these methods exceeds the scope of this work, the verification of the proposed model needs to be performed at a sufficient level.

The set of verification scenarios is prepared to verify the behaviour of the model within various simulated conditions. The conditions vary due to definition of the approach procedure, irregularities of the navigation signal, weather conditions or due to other causes not incorporated within the scope of this work.

The functionality of the developed model is verified against established goals. One group of the goals for each precise approach category as a window is specified in $\mathrm{Ta}$ ble 1 . The other group is defined using the navigation performance parameters below. Simulations are performed in the scenarios which are designed based on verification objectives. The outcomes of simulations are statistically analysed and further compared with the value ranges expected by the goals.

The verification objectives determined based on the flight manual of the L-410 aircraft (LET, 1996) are as follows:

1. Approach on curve. The planned curved trajectory is defined within the localizer plane;

2. Glide slope angles in an interval from 2.75 to 3.77 degrees with a specific interest in the value of 3.00;

Table 2. System of evaluation scenarios

\begin{tabular}{|c|c|c|}
\hline $\begin{array}{l}\text { Scenario } \\
\text { number }\end{array}$ & $\begin{array}{l}\text { Navigation and } \\
\text { control }\end{array}$ & Scenario specific focus \\
\hline 1 & \multirow[t]{4}{*}{ Lateral } & None \\
\hline 2 & & Curve \\
\hline 3 & & Drift \\
\hline 4 & & Navigation signal dropout \\
\hline 5 & \multirow[t]{5}{*}{ Longitudinal } & Glide slope angle of 2.75 degrees \\
\hline 6 & & Glide slope angle of 3.00 degrees \\
\hline 7 & & Glide slope angle of 3.77 degrees \\
\hline 8 & & Head wind \\
\hline 9 & & Navigation signal dropout \\
\hline
\end{tabular}

3. Dropout of navigation signal for 2 seconds;

4. Wind drift of $10 \mathrm{~m} / \mathrm{s}$;

5. Head wind of $5 \mathrm{~m} / \mathrm{s}$.

The selected scope of the approach starts in the final approach fix (FAF) point and ends by transferring to the flare. In the event that the FAF distance from the runway is $9630.4 \mathrm{~m}$ (5.2 nautical miles) and the threshold height for the start of flare is $3 \mathrm{~m}$. The initial speed is $250 \mathrm{~km} / \mathrm{h}$ and the target speed at the flare height is $155 \mathrm{~km} / \mathrm{h}$. The set of verification scenarios based on the verification objectives is listed in Table 2.

The designed model proposed for verification includes a set of input signals dedicated for the purpose of simulation execution in the environment configured for the given scenario. The following are the signals for lateral aircraft control:

a) Reference trajectory;

b) Drift;

c) Validity flag of the navigation signal;

d) Navigational signal trimming.

Longitudinal scenarios are configured using the following model inputs:

a) Glide slope angle;

b) Headwind;

c) Validity flag of the navigation signal;

d) Navigational signal trimming.

\subsection{Evaluation methodology}

The verification approach is mostly determined by the selected method of the modelling of the studied systems and processes. The attempt for the development of the functional prototype of the control system requires the generation of stochastic data and simulation executions. Guidance for the statistical analysis of the data which resulted from the executions are provided by the $\mathrm{PBN}$ principles stated in the ICAO Document 9613 (ICAO, 2008) which govern APV approaches.

Requirements provided on the position of the aircraft are expressed in the following statistical manner:

1. The difference of the actual position of the aircraft from its planned position is evaluated;

2. Standard deviation of the difference should not exceed the distance given by the selected navigation category;

3. The maximum of the difference should not exceed double the given distance.

\subsection{Goals}

The navigation goals developed for the verification purposes of the presented solution are established as a combination of two concepts: of the navigation using the ILS system and of the performance-based navigation. The first concept allows to prove that the existing navigation requirements which currently ensure safety of the approach procedure are fulfilled. The corresponding goals are specified in Table 1. 
The specification of the navigation performance to be achieved by the APV approach is provided within the scope of PBN by specified required navigation performance (RNP). The RNP for the approaches provides the requirements on both lateral and vertical navigation (ICAO, 2009) as so as for recommended RNP 0.003/15 (FAA, 2006) for approach procedure being studied within scope of this work. Detailed statistical characteristics are derived as follows:

1. Standard deviation of the difference in the lateral position within $5.556 \mathrm{~m}$;

2. Standard deviation of the difference in altitude within $3.048 \mathrm{~m}$;

3. Maximum difference in the lateral position of $11.112 \mathrm{~m}$;

4. Maximum difference in the altitude of $6.096 \mathrm{~m}$.

\section{Results}

The evaluation of outcomes of the model simulations executed for the scenarios per verification objectives is presented in Figure 6 and Figure 7. The first four scenarios are executed with the lateral model while the other five are executed with the longitudinal one. The displayed results of verification are combined for both horizontal and vertical directions, i.e. for distance from the planned trajectory in the direction perpendicular to the localizer plane and at a height above the runway, respectively.

Figure 6 shows the difference of the actual aircraft position from the centre of the approach window defined in Table 1 compared to definitions of the window sizes for the equivalent ILS categories. The developed model controls the simulated aircraft down to the reference height of the approach window so that the position of the aircraft is within the window for all scenarios.

Similarly, goals for the distance and for the height are combined within the chart in Figure 7. Both maxima of values and the standard deviation of values during the simulated approach procedure are shown along with the respective RNP required values. Again, the model fulfills the RNP requirements for the executed scenarios.

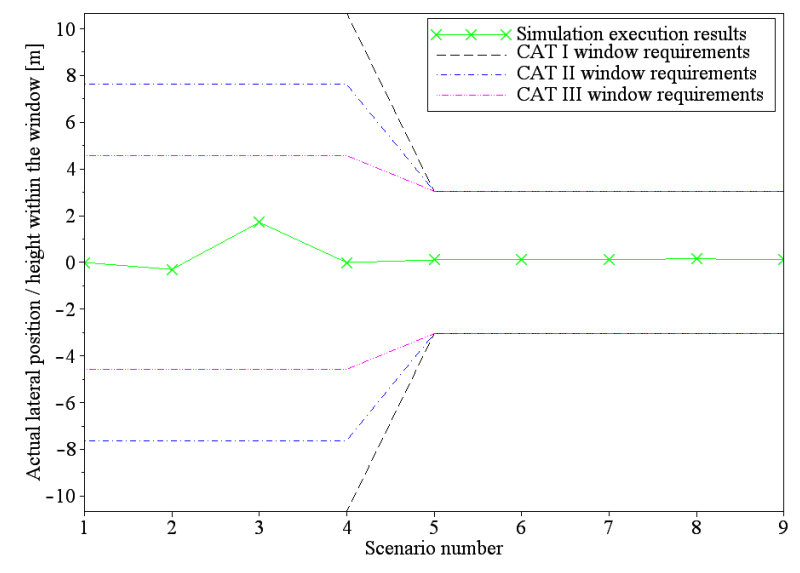

Figure 6. Comparison of actual aircraft position within the windows against their defined sizes

\section{Conclusions}

The aim of this work was to follow up on the statistical analysis of real EGNOS measurement to exercise these data against a simulated process of the precise instrument LPV approach. Focus was given to the fact that, compared to ILS, navigational data are received as discrete samples. The other area of focus, besides studying the behaviour of the model control in altitude, was to attempt to challenge the proposed automated control system within the simulated environment of approach on the curve which is a huge advantage of LPV approaches versus ILS ones.

Model-based development was selected as a platform for the study. In this way, it was possible to design and develop a functional prototype of the automated control system. Scilab 5.4.1 was used to completely cover modelling, simulation, data processing and data analysis of the effort while using graphical and scripting features of the environment. The presented work demonstrates how complex systems and processes can be modelled and simulated in an integrated and consistent manner. Further expansion of the model was considered during the implementation process so that the final architecture of the model is readable, and the modules are kept replaceable.

Navigation performance goals for the simulation environment were defined based on performance-based navigation guidelines. Analysis of simulation executions of the model proves that it is possible to control the aircraft within RNP requirements and requirements equivalent to the ILS CAT I, II and III approaches below the decision height of 60 metres. The main assumptions of the achieved results are that other sensors are also utilized during the approach and that the verification is solely built on the laboratory experiment. The ILS CAT III operation is in question as far as the flare was not analysed.

The priority of the work was to develop a functional prototype of the automated control system operation with EGNOS navigational signal inputs which implies following the most significant limitations of the work. Analysis was performed exclusively in the simulated environment

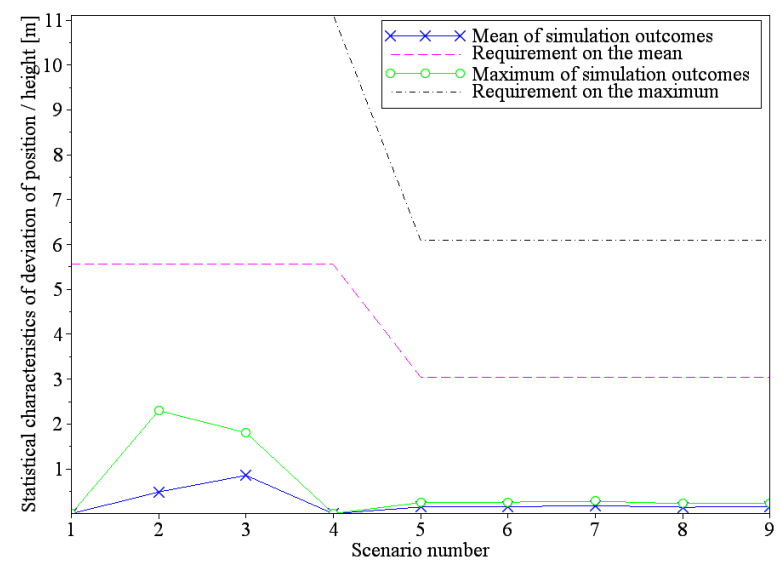

Figure 7. Comparison of statistical characteristics extracted from simulation outcomes against values required by the goals 
and solely with the use of stochastically generated data. The control model was based solely on EGNOS data; no other sensors were considered. The elaboration on the modules of the system was performed up to the simplest level which supported the experiment, but not in more detail.

The presented work covers several topics of modelling and simulation areas and so it can easily be considered as a starting point for consequent studies. The scope may be expanded in replacing the aircraft dynamic module to examine the behaviour of other aircraft in a wider set of scenarios. An additional focus may also be given to improvements of the navigation filter, including other sensors into a control mechanism, a broader study of the initialization conditions of simulation and a specific focus on drift impact on the controlled approach process.

\section{Disclosure statement}

I am not aware of any competing financial or personal interests from other parties. Regarding professional interests, I have been involved in projects in the past where data processing and simulations were used. However, none of the information except for the professional experience gained was used within the scope of this work.

\section{References}

Antemijczuk, O., Sokolowska, D., \& Cyran, K. A. (2012). Integration of the MS ESP flight simulator with GNSS-based guidance system. Recent Advances in Systems Science and Mathematical Modeling. http://www.wseas.us/e-library/conferences/2012/Paris/MATHSYS/MATHSYS-39.pdf

Balate, J. (2003). Automaticke rizeni [Automatic Control]. BEN.

Beldjilali, B., Kahlouche, S., \& Tabti, l. (2020). Assessment of EGNOS performance for civil aviation flight phase in the edge coverage area. International Journal of Aviation, Aeronautics, and Aerospace, 7(3), 1.

https://doi.org/10.15394/ijaaa.2020.1479

Cook, M. V. (2012). Flight dynamics principles: a linear systems approach to aircraft stability and control. Butterworth-Heinemann.

CTU. (2015, September 15). Predmet A3M35SRL. Systemy rizeni letu [Lecture A3M35SRL. Flight Control Systems]. Czech Technical University in Prague. https://moodle.fel.cvut.cz/lo$\mathrm{cal} /$ kos/pages/course/info.php?id=1712

EGNOS. (2002). Trials in Nice (2004, October 21). European Geostationary Navigation Overlay Service. http://www.eurocontrol.fr/Newsletter/2002/November/EGNOS/Version\%20 $0 \_4 \mathrm{~b} / \mathrm{NewsletterArticle} 4 \mathrm{~b} . \mathrm{htm}$

EGNSSA. (2020, Oct 13). Aviation. European Global Navigation Satellite Systems Agency. https://egnos-user-support.essp-sas. eu/new_egnos_ops/segments/aviation

FAA. (2006). Advisory Circular AC 120-29A, Criteria for Approval of Category I and Category II Weather Minima for Approach. Federal Aviation Administration. U.S. Department of Transportation. https://www.faa.gov/documentLibrary/ media/Advisory_Circular/AC120-29A.pdf

Fellner, A. (2011, October). LPV flight trials in Poland. In International Conference on Transport Systems Telematics (pp. 320329). Springer. https://doi.org/10.1007/978-3-642-24660-9_37
Grunwald, G., Ciecko, A., \& Tanajewski, D. (2019, January). Analysis of Applying the EGNOS System in APV-1 and LPV200 Operations. In IOP Conference Series: Earth and Environmental Science. IOP Publishing.

https://doi.org/10.1088/1755-1315/221/1/012075

ICAO. (2018). Annex 6 - Operation of Aircraft. Part I International Commercial Air Transport - Aeroplanes. International Civil Aviation Organisation. https://store.icao.int/en/annex6-operation-of-aircraft-part-i-international-commercial-airtransport-aeroplanes

ICAO. (2016). Annex 10 - Aeronautical Telecommunications. Volume II: Communication Procedures. International Civil Aviation Organisation. https://store.icao.int/en/annex-10-aeronautical-telecommunications-volume-ii-communicationprocedures-including-those-with-pans-status

ICAO. (2020). Doc 8168. Procedures for Air Navigation Services Aircraft Operations - Volume II Construction of Visual and Instrument Flight Procedures. International Civil Aviation Organisation. https://store.icao.int/en/procedures-for-airnavigation-services-pans-aircraft-operations-volume-ii-construction-of-visual-instrument-flight-procedures-doc-8168

ICAO. (2008). Doc 9613. Performance-based Navigation (PBN) Manual. International Civil Aviation Organisation. https:// www.icao.int/sam/documents/2009/samig3/pbn\%20manual\%20-\%20doc\%209613\%20final\%205\%2010\%2008\%20 with\%20bookmarks1.pdf

ICAO. (2009). Doc 9905. Required Navigation Performance Authorization Required (RNP AR). International Civil Aviation Organisation. https://www.icao.int/meetings/pbn-symposium/documents/9905_cons_en.pdf

ICAO. (2012). Doc 9849. Global Navigation Satellite System (GNSS) Manual. International Civil Aviation Organisation. https://www.icao.int/Meetings/anconf12/Documents/ Doc.\%209849.pdf

Krasuski, K., \& Wierzbicki, D. (2020). Monitoring aircraft position using EGNOS data for the SBAS APV approach to the landing procedure. Sensors, 20(7), 1945.

https://doi.org/10.3390/s20071945

LET. (1996). Airplane Flight Manual for the L 410 UVP - E20. Book 1 (Sections 0 to 5). LET Kunovice.

Meloun, M., \& Militky, J. (2002). Kompendium statistickeho zpracovani dat. Metody a resene ulohy vcetne CD [Compendium of Statistic Data Processing. Methods and Solved Examples Including CD]. Academia.

Ptacek, P. (2014). Vykonnost sluzby GNSS pro aplikace prostorove navigace civilniho letectvi $v$ CR [The Performance of GNSS Services for the Area Navigation Applications of Civil Aviation in CR] [Doctoral dissertation, Brno University of Technology]. Brno, Czech Republic.

RTCA. (2012). DO-178C, Software Considerations in Airborne Systems and Equipment Certification. Radio Technical Commission for Aeronautics.

Scilab. (2020, May 2). Design of Experiments and optimization of aircraft design. ESI Group. https://www.scilab.org/use-cases/ design-of-experiments-and-optimization-of-aircraft-design/

Sorenson, H. W. (1985). Kalman filtering: theory and application. IEEE.

Vassilev, B., \& Vassileva, B. (2012). EGNOS performance before and after applying an error extraction methodology. Annual of Navigation, 19(2), 121-130. https://doi.org/10.2478/v10367-012-0022-8

Vencius, V. (2013). Application of SBAS and ABAS Systems in Lithuanian Aviation. Aviation Technologies, 1(2). 


\section{Appendix A}

The polynomial function is used as a smoother which interpolates discrete filtered navigation data by continuous signal. Requirement by the control system including proportional and derivative components are the continuity of the signal and its first derivative. The extrapolation of the continuous signal was selected as based on the last two known pairs of position and its change. It ensures the smallest transport delay through the smoother. In such a case, an independent variable of the polynomial function can be defined to have discrete data known as pairs of $f(0), f^{\prime}(0)$ and $f(1), f^{\prime}(1)$ if the second pair represents more recent data. Such a polynomial function composes sextic spline for each interval starting at the time of every new filtered discrete pair sample while the requirement on continuity is fulfilled. After considering derivatives of the spline, three unknown coefficients $a, b$, and $c$ need to be determined for each new discrete sample:

$f(t)=a t^{5}+b t^{4}+c t^{3}+f^{\prime \prime}(0) t^{2} / 2+f^{\prime}(0) t+f(0)$.

It can be derived that:

$$
\left[\begin{array}{l}
a \\
b \\
c
\end{array}\right]=\left[\begin{array}{ccc}
6 & -3 & 1 / 2 \\
-15 & 7 & -1 \\
10 & -4 & 1 / 2
\end{array}\right]\left[\begin{array}{l}
\alpha \\
\beta \\
\gamma
\end{array}\right],
$$

when the following substitutions are used:

$$
\begin{aligned}
& \alpha=f(1)-f(0)-f^{\prime}(0)-f^{\prime \prime}(0) / 2, \\
& \beta=f^{\prime}(1)-f^{\prime}(0)-f^{\prime \prime}(0), \\
& \gamma=f^{\prime \prime}(1)-f^{\prime \prime}(0) .
\end{aligned}
$$

Implementation of the smoother in Scilab can be further simplified by putting $f^{\prime \prime}(0)=f^{\prime \prime}(1)=0$ as far as the estimation of the second derivative of the position is not calculated in the selected Kalman filter design. The resulted implementation as the script lines in Scilab is following.

$-\mathrm{dt}=\mathrm{t}-\mathrm{z}(1)$;

$-\mathrm{aa}=\mathrm{z}(2)$;

$-\mathrm{bb}=\mathrm{z}(3)$;

$-\mathrm{cc}=\mathrm{z}(4)$;

- ee $=z(5)$;

$-\mathrm{ff}=\mathrm{z}(6)$;

$-\mathrm{y} 10=\left(((\mathrm{aa} \cdot \mathrm{dt}+\mathrm{bb}) \cdot \mathrm{dt}+\mathrm{cc}) \cdot \mathrm{dt}^{\wedge} 2+\mathrm{ee}\right) \cdot \mathrm{dt}+\mathrm{ff} ;$

$-\mathrm{y} 20=((5 \cdot \mathrm{aa} \cdot \mathrm{dt}+4 \cdot \mathrm{bb}) \cdot \mathrm{dt}+3 \cdot \mathrm{cc}) \cdot \mathrm{dt} \wedge 2+\mathrm{ee} ;$

$-\mathrm{z}(1)=\mathrm{t}$;

- alfa $=\mathrm{u} 1-\mathrm{y} 10-\mathrm{y} 20$;

- betax $=\mathrm{u} 2-\mathrm{y} 20$;

$-z(2)=6 \cdot \alpha-3 \cdot \beta$

$-z(3)=-15 \cdot \alpha+7 \cdot \beta$

$-z(4)=10 \cdot \alpha-4 \cdot \beta$;

$-\mathrm{z}(5)=\mathrm{y} 20$;

$-\mathrm{z}(6)=\mathrm{y} 10$;

The spline coefficient calculation (aa to ff) is based on the new sample $\mathrm{u} 1$ and its derivative $\mathrm{u} 2$ and on previous sample y10 and its derivative y 20 memorized from the previous coefficient set.

\section{Appendix B}

Implementations of the evaluated model for control of the aircraft movement during the approach in the lateral and longitudinal directions are shown in Figure 8 and Figure 9, respectively.

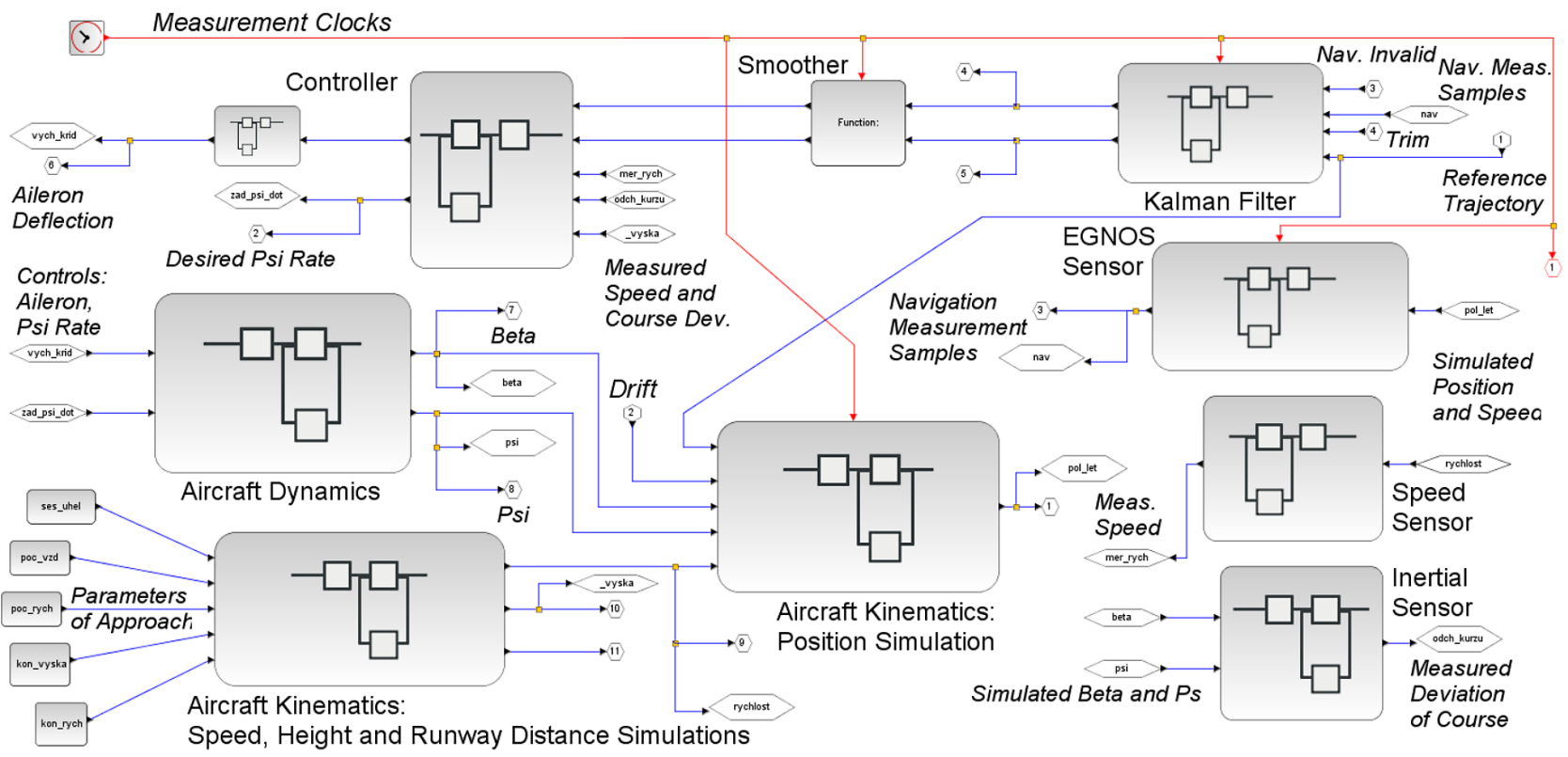

Figure 8. Overview of automated control system model and simulation of the approach process in the lateral direction 


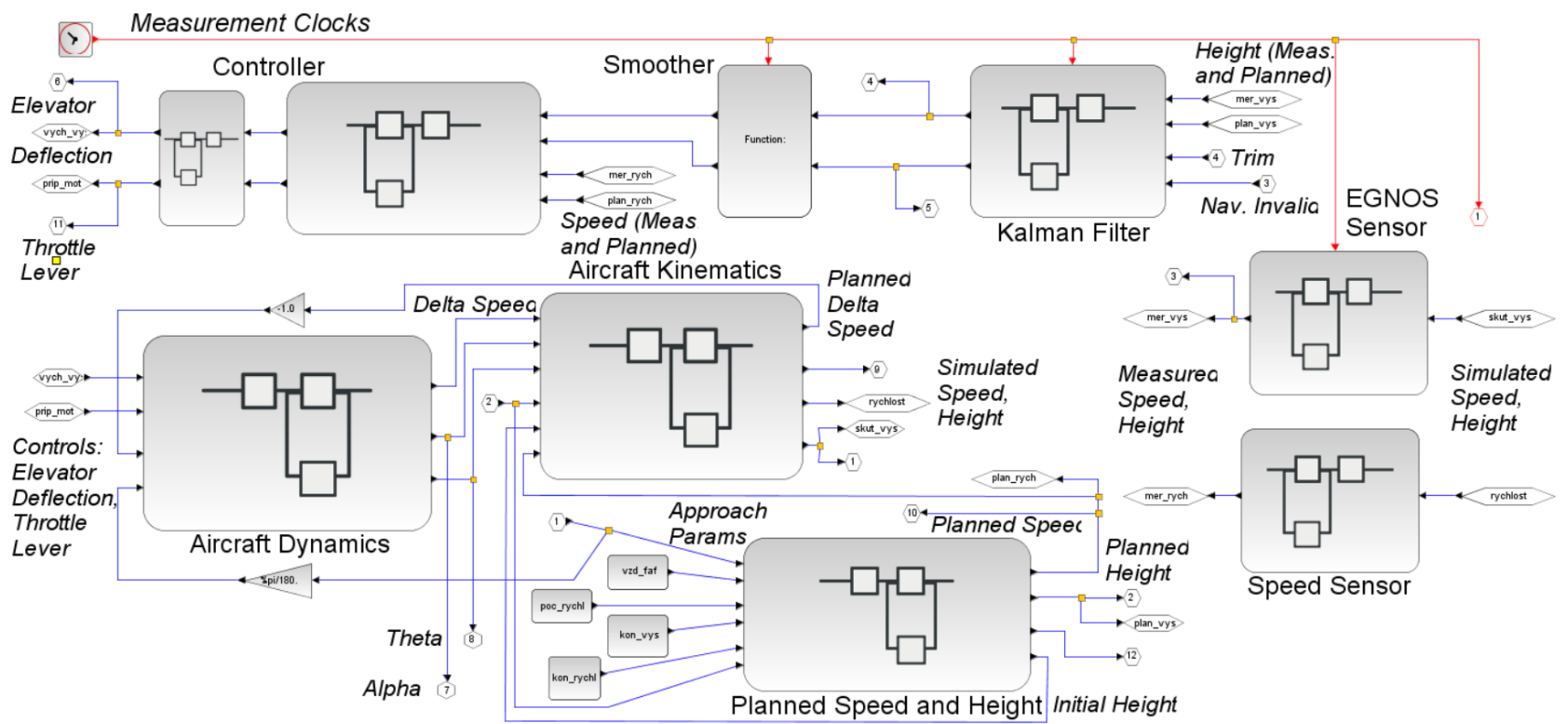

Figure 9. Overview of automated control system model and simulation of the approach process in the longitudinal direction

\section{Notations}

\section{Variables and functions}

$A$ - State matrix of a state space linear system;

$a$ - The fifth order coefficient of sextic spline;

$a$ - Main axis of hyperbolic approach curve;

$B$ - Input matrix of a state space linear system;

$b$ - The fourth order coefficient of sextic spline;

C - Output matrix of a state space linear system;

$c$ - The third order coefficient of sextic spline;

$D$ - Feedthrough matrix of a state space linear system;

$F$ - Matrix of the linear Kalman filter inner evolution model;

$F^{\prime}$ - Transposition of matrix $F$;

$f(t)$ - Sextic spline function in time;

$f^{\prime}(t)$ - The first derivative of the spline function;

$f^{\prime \prime}(t)$ - The second derivative of the spline function;

$H$ - Matrix of projection from the Kalman filter state space to the measurement state space;

$I$ - Identity matrix;

$I_{j k}$ - Elements of moments of inertia tensor of the aircraft;

$K$ - Kalman filter gain;

$L_{Y}$ - The first element of force momentum vector of origin $\mathrm{Y}$ which has an effect on the aircraft body;

$m$ - Aircraft weight;

$M_{Y}$ - The second element of force momentum vector of origin $\mathrm{Y}$ which has an effect on the aircraft body;

$m$ - Offset of the hyperbolic curve center along the approach axis;

$N_{Y}$ - The third element of force momentum vector of origin $\mathrm{Y}$ which has an effect on the aircraft body;

$n$ - Offset of the hyperbolic curve center with respect to the localizer plane;
$P$ - The first element of aircraft body angular speed vector;

$\dot{P}$ - The first time derivative of vector element $P$;

$Q$ - Noise covariance matrix of the Kalman filter inner evolution model;

$Q$ - The second element of aircraft body angular speed vector;

$R$ - Covariance matrix of errors of measurements on the Kalman filter input;

$R$ - The third element of aircraft body angular speed vector; $S_{n \mid n-1}$ - Estimated covariance matrix of Kalman filter state errors based on previous filtration step;

$t$ - Time;

$U$ - The first element of aircraft body speed vector;

$u$ - Control vector of a state space linear system;

$V$ - The second element of aircraft body speed vector;

$v$ - Aircraft height above the runway;

$W$ - The third element of aircraft body speed vector;

$X_{X}$ - The first element of force vector of origin $\mathrm{X}$ which has an effect on the aircraft body;

$x_{n-1 \mid n-1}$ - Vector of Kalman filter states in the previous filtration step;

$x_{n \mid n-1}$ - Vector of estimated filter states based on the previous filtration step;

$x$ - State vector of a state space linear system;

$\dot{x}$ - Time derivative of the state vector of a state space linear system;

$(x)_{E}$ - Estimation of $\mathrm{x}$ based on its measurements;

$x$ - Lateral position of the aircraft with respect to the localizer plane;

$x_{0}$ - Actual lateral position of the aircraft with respect to the localizer plane;

$Y_{X}$ - The second element of force vector of origin $\mathrm{X}$ which has an effect on the aircraft body;

$y$ - Output vector of a state space linear system; 
$Z_{X}-$ The third element of force vector of origin $\mathrm{X}$ which has an effect on the aircraft body;

$z$ - Distance of the aircraft from the runway along the approach axis;

$z_{0}$ - Actual distance of the aircraft from the runway along the approach axis;

$z_{F A F}$ - Distance of the final approach fix from the runway;

$\alpha$ - Aircraft angle of attack;

$\alpha$ - Substitution used for derivation of spline coefficients;

$\beta$ - Aircraft side slip angle;

$\beta$ - Substitution used for derivation of spline coefficients;

$\gamma$ - Aircraft roll angle;

$\dot{\gamma}$ - Aircraft roll rate;

$\gamma$-Substitution used for derivation of spline coefficients;

$\Delta t$ - Measurement sampling time period;

$\Delta x$ - The difference of the actual and planned position of the aircraft;

$\Delta y$ - The second estimated state of Kalman filter;

$\eta$ - Aircraft elevator position;

$\vartheta$ - Aircraft pitch angle;

$\dot{\vartheta}$ - Aircraft pitch rate;

$\xi$ - Aircraft aileron position; $\varsigma$ - Aircraft rudder position;

$\tau$ - Aircraft thrust lever position;

$\psi$ - Aircraft yaw angle;

$\dot{\psi}$ - Aircraft yaw rate;

$\psi_{0}$-Aircraft yaw corresponding to hyperbolic curve asymptote;

$\dot{\psi}_{c}$ - Change of aircraft yaw along the curve;

0 - Matrix of zero elements.

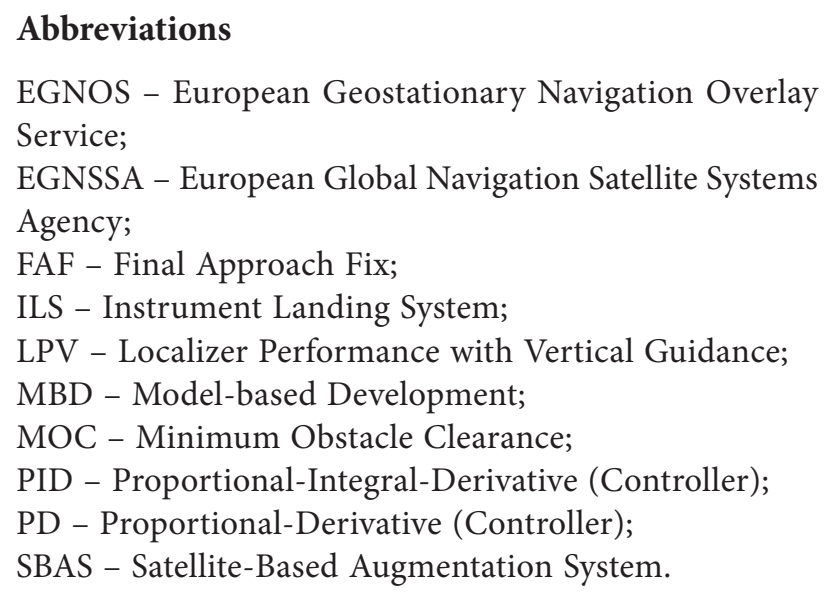

